OPEN ACCESS

Edited by:

Gentaro Iribe,

Asahikawa Medical University, Japan

Reviewed by:

lan Findlay,

ERL7003 Laboratoire Signalisation et

Transports Ioniques Membranaires

(STIM), France

Tong Liu,

Tianjin Medical University, China

${ }^{*}$ Correspondence:

Alexey V. Glukhov

aglukhov@medicine.wisc.edu

Specialty section:

This article was submitted to

Cardiac Electrophysiology,

a section of the journal

Frontiers in Physiology

Received: 30 November 2019

Accepted: 02 March 2020

Published: 26 March 2020

Citation:

Egorov W, Rosenshtraukh LV and Glukhov AV (2020)

Arrhythmogenic Interaction Between

Sympathetic Tone and Mechanical

Stretch in Rat Pulmonary Vein

Myocardium. Front. Physiol. 11:237.

doi: 10.3389/fphys.2020.00237

\section{Arrhythmogenic Interaction Between Sympathetic Tone and Mechanical Stretch in Rat Pulmonary Vein Myocardium}

\author{
Yuriy V. Egorov ${ }^{1}$, Leonid V. Rosenshtraukh ${ }^{1}$ and Alexey V. Glukhov ${ }^{2 *}$ \\ ${ }^{1}$ Laboratory of Heart Electrophysiology, Russian Cardiology Research Centre, Institute of Experimental Cardiology, Moscow, \\ Russia, ${ }^{2}$ Department of Medicine, School of Medicine and Public Health, University of Wisconsin-Madison, Madison, WI, \\ United States
}

Rapid firing from pulmonary veins (PVs) frequently initiates atrial fibrillation, which is a common comorbidity associated with hypertension, heart failure, and valvular disease, i.e., conditions that pathologically increase cardiomyocyte stretch. Autonomic tone plays a crucial role in PV arrhythmogenesis, while its interplay with myocardium stretch remains uncertain. Two-microelectrode technique was used to characterize electrophysiological response of Wistar rat PV to adrenaline at baseline and under mild (150 mg of applied weight that corresponds to a pulmonary venous pressure of $1 \mathrm{mmHg})$ and moderate $(10 \mathrm{~g}, \sim 26 \mathrm{mmHg})$ stretch. Low concentrations of adrenaline $(25-100 \mathrm{nmol} / \mathrm{L})$ depolarized the resting membrane potential selectively within distal PV (by $26 \pm 2 \mathrm{mV}$ at baseline, by $18 \pm 1 \mathrm{mV}$ at $150 \mathrm{mg}$, $P<0.001$, and by $5.9 \pm 1.1 \mathrm{mV}$ at $10 \mathrm{~g}, P<0.01$ ) suppressing action potential amplitude and resulting in intra-PV conduction dissociation and rare episodes of spontaneous activity (arrhythmia index of $0.4 \pm 0.2$, NS vs. no activity at baseline). In contrast, $1-10 \mu \mathrm{mol} / \mathrm{L}$ of adrenaline recovered intra-PV propagation. While mild stretch did not affect PV electrophysiology at baseline, moderate stretch depolarized the resting potential within distal PV $(-56 \pm 2 \mathrm{mV}$ vs. $-82 \pm 1 \mathrm{mV}$ at baseline, $P<0.01$ ), facilitated the triggering of rapid $P V$ firing by adrenaline (arrhythmia index: $4.4 \pm 0.2$ vs. $1.3 \pm 0.4$ in unstretched, $P<0.001$, and $1.7 \pm 0.8$ in mildly stretched preparations, $P<0.005$, at $10 \mu \mathrm{mol} / \mathrm{L}$ adrenaline) and induced frequent episodes of potentially arrhythmogenic atrial "echo" extra beats. Our findings demonstrate complex interactions between the sympathetic tone and mechanical stretch in the development of arrhythmogenic activity within PVs that may impact an increased atrial fibrillation vulnerability in patients with elevated blood pressure.

\footnotetext{
Keywords: pulmonary veins, stretch, arrhythmia, mechano-electrical response, adrenalin
} 


\section{INTRODUCTION}

Focal electrical activity from pulmonary veins (PVs) frequently initiates atrial fibrillation (Haissaguerre et al., 1998; Chen et al., 1999), which is a common comorbidity associated with hypertension, heart failure, and valvular disease (Schotten et al., 2011). These conditions cause hemodynamic atrial overload resulting in pathologically increased cardiomyocyte stretch and facilitates the arrhythmogenic activity from PVs (Tsao et al., 2001). In the setting of atrial dilatation, mechanoelectrical feedback has been linked to the development of ectopic beats that trigger atrial fibrillation. However, the precise mechanisms remain poorly understood.

We (Egorov et al., 2015, 2019) and others (Arora et al., 2003; Walters et al., 2014; Pasqualin et al., 2018) have demonstrated a significant electrophysiological heterogeneity of myocardial cells within PVs, which may form a functional substrate for focal activity and echo extra beats. This was linked to a distinct ionic channel and $\mathrm{Ca}^{2+}$-handling gene repertoire in PVs that would underlie their distinct response to proarrhythmic stimuli. Our recent study revealed a tension-dependent stretch-induced depolarization of the resting membrane potential (RP) within the distal part of rat $\mathrm{PV}$, which decreased action potential (AP) amplitude (APA) and triggered conduction discontinuities and both ectopic and reentrant activities within the vein (Egorov et al., 2019). One may suggest that such stretch-induced arrhythmogenesis would significantly interfere with autonomic tone that plays a crucial role in triggering rapid firing from PVs (Patterson et al., 2005; Doisne et al., 2009). It was shown that the differences in the RP and reaction to adrenergic stimulation between the PV and the left atrium leads to automatic electrical activity occurring specifically in PV (Doisne et al., 2009). Indeed, PV myocytes demonstrated a low density of the $\mathrm{K}_{\mathrm{ir}} 2 . \mathrm{x}$ channels and the RP stabilizing inwardly rectifying current $I_{\mathrm{K} 1}$ (Melnyk et al., 2005; Tsuneoka et al., 2017), increased resting $\mathrm{Na}^{+}$permeability (Malecot et al., 2015), and enhanced chloride conductance (Okamoto et al., 2014).

In this study, we hypothesized that myocardial stretch would facilitate the development of arrhythmogenic ectopic activity induced by sympathetic stimulation in the PV. To test this, two-microelectrode technique was used to characterize a regionspecific electrophysiological response of rat PV myocardium to low $(25-100 \mathrm{nmol} / \mathrm{L})$ and high $(1-10 \mu \mathrm{mol} / \mathrm{L})$ concentrations of adrenaline at baseline and under mild (150 $\mathrm{mg}$ of applied weight, which corresponds to a PV pressure of $1 \mathrm{mmHg}$ ) and moderate $(10 \mathrm{~g}, \sim 26 \mathrm{mmHg})$ stretch.

\section{MATERIALS AND METHODS}

\section{Animals and Preparations}

All methods and protocols used in these studies have been approved by the Animal Care and Use Committee of the Cardiology Research Center (Moscow, Russia) following the Guidelines for Care and Use of Laboratory Animals published by the National Institutes of Health (NIH) (publication no. 85-23, revised 1996). All animals used in this study received humane care in compliance with the Guide for the Care and Use of Laboratory Animals. Adult (8- to 12-month-old) Wistar rats $(n=7)$ of both sexes were used. Rats were anesthetized with urethane $2 \mathrm{~g} / \mathrm{kg}$ with heparin $(0.2 \mathrm{U})$, and the loss of pain reflex was evaluated to assure the appropriate level of anesthesia. The isolated PV preparation was performed as described previously (Egorov et al., 2015, 2019). Briefly, after mid-sternal incision, the heart with lungs was removed and placed in oxygenated $\left(95 \% \mathrm{O}_{2}, 5 \% \mathrm{CO}_{2}\right)$ room-temperature Tyrode solution of the following composition (in mmol/L): $118 \mathrm{NaCl}, 1.8 \mathrm{CaCl}_{2}, 1.2$ $\mathrm{MgCl}_{2}, 4.7 \mathrm{KCl}, 1.2 \mathrm{NaH}_{2} \mathrm{PO}_{4}, 25 \mathrm{NaHCO}_{3}$, and 11 glucose $(\mathrm{pH}=7.35 \pm 0.05)$. The left atrium (LA) together with the LA appendage and PV region was dissected from the ventricles, right atrium, and interatrial septum. The preparation then was placed in a tissue bath $(2.5 \mathrm{ml})$ and continuously superfused with oxygenated Tyrode's solution $(18 \mathrm{ml} / \mathrm{min})$ at $37 \pm 0.5^{\circ} \mathrm{C}$. Central PV was cleaned from fat and lung tissues, beside the LA, lanced and then positioned on a thin coat of silicon on the bottom of a tissue bath with the endocardial side facing upward. The pacing electrode was placed on the edge of the left atrial appendage. A small portion of the end-distal part of the PV was not cut open and was used to weave a silk suture (4-0) with a weight applied.

\section{Microelectrode Recordings}

Transmembrane potentials were simultaneously recorded from the endocardial surface of the distal $\left(\mathrm{PV}_{\mathrm{dis}}\right)$ and the ostial $\left(\mathrm{PV}_{\text {ost }}\right)$ parts of the PV (Egorov et al., 2019) using two glass microelectrodes filled with $3.0 \mathrm{mmol} / \mathrm{L} \mathrm{KCl}$ (tip resistance, $\sim 10$ $40 \mathrm{M} \Omega$ ) and connected to high-input impedance amplifiers (WPI model KS-701, World Precision Instruments, New Haven, CT, United States). Microelectrodes were stably maintained within the tissue during all the measurements within each experimental condition (unstretched, $150 \mathrm{mg}$ and stretch, $10 \mathrm{~g}$; Figure 1). In some preparations, microelectrode stability was lost during 1-h stretch applications (i.e., between experimental conditions), and repenetration in nearby tissue was performed. Transmembrane potential signals were recorded, digitized (sampling rate of $5 \mathrm{kHz}$ ) using analog-digital converter (E-154, L-Card, Moscow, Russia), and then saved on a computer for offline analysis as described previously (Egorov et al., 2015). To characterize electrophysiological properties of the PV myocardium, RP and APA were measured during S1S1 $=300 \mathrm{~ms}$ pacing. The pacing current was at least $2 \times$ the pacing threshold.

\section{Experimental Protocol}

Experimental protocol is shown schematically in Figure $\mathbf{1}$. Measurements were performed 40-60 min after the isolation procedure. Three adrenaline concentrations $(25-100 \mathrm{nmol} / \mathrm{L}$, 1 and $10 \mu \mathrm{mol} / \mathrm{L}$ ) were tested at baseline (no stretch applied), under mild stretch (150 mg of weight applied, which approximately corresponds to a physiological pulmonary venous pressure of $1 \mathrm{mmHg}$ calculated as applied weight $\times$ gravity constant/cross-section area of the PV preparation) and under moderate stretch (10 g of applied weight corresponding approximately to $26 \mathrm{mmHg}$ of pulmonary venous pressure) as described previously (Egorov et al., 2019). For each condition, 


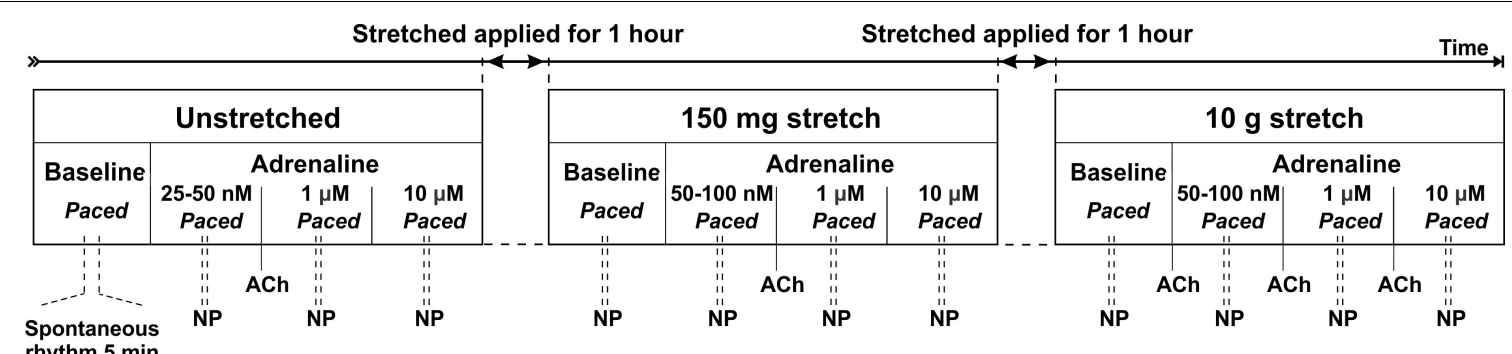

Membrane resting potential rhythm 5 min
(no pacing, NP)
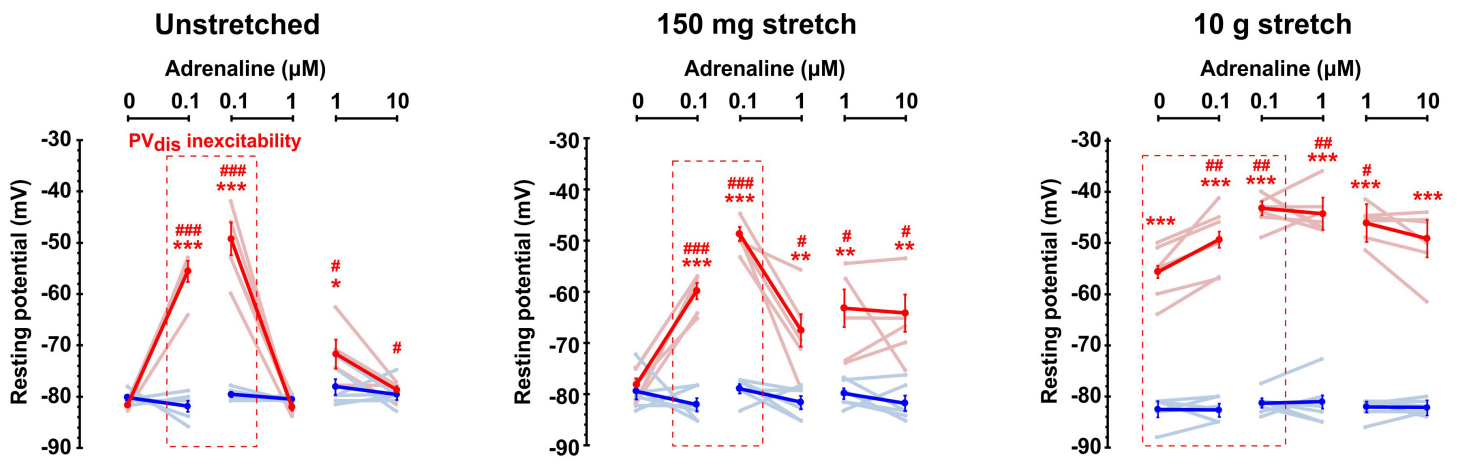

\section{Action potential amplitude}
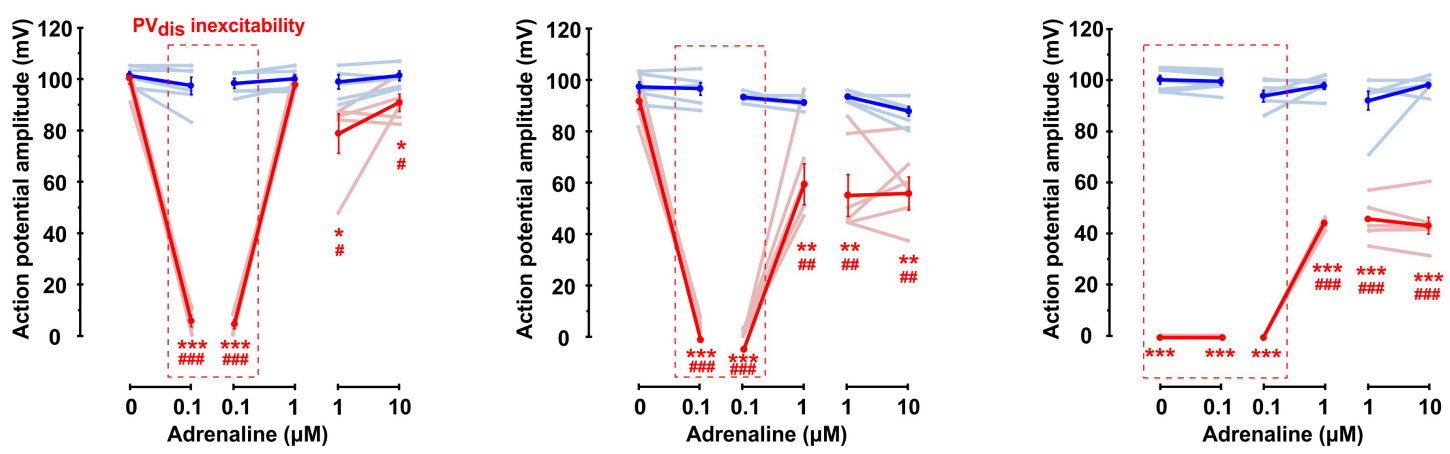

FIGURE 1 | Complex interactions between sympathetic tone and mechanical stretch in rat pulmonary vein (PV) myocardium. Top: Experimental protocol for testing different levels of sympathetic stimulation in unstretched and stretched PV preparations. Physiological status of $P V_{\text {dis }}$ inexcitability induced be either low concentrations of adrenaline (25-100 nmol/L), or pathological stretch (10.5 g) was tested by a brief application of acetylcholine (ACh), which was administered to hyperpolarize the RP and recover $\mathrm{PV}_{\text {dis }}$ excitability and then washed out to continue experimental protocol. All measurements were performed during constant left atrial pacing, except brief (5-10 min) periods when pacing was stopped (no pacing, NP), and spontaneous rhythm was recorded if present. Below, changes in the resting membrane potential (middle panels) and action potential amplitude (low panels) are shown for distal (red traces) and ostium (blue traces) PV regions at baseline (no adrenaline applied) and under low (25-100 nmol/L) and high (1-10 $\mu \mathrm{mol} / \mathrm{L})$ concentrations of adrenaline measured in unstretched and stretched (150 mg and $10 \mathrm{~g}$ ) preparations. Changes are shown for individual rats (light blue and light red lines) as well as mean \pm SEM (solid lines). Red dashed box indicates the presence of $P V_{\text {dis }}$ inexcitability. $N=6-7$ rats. ${ }^{\star} P<0.05$, ${ }^{\star \star} P<0.01$, ${ }^{\star \star \star} P<0.001$ for $P V_{\text {dis }} V_{s}$. $P V_{\text {ost }}$; ${ }^{\#} P<0.05$, ${ }^{\# \#} P<0.01$, ${ }^{\# \# \#} P<0.001$ within the same group vs. baseline by repeated measurements two-way ANOVA with Bonferroni correction.

we first tested nanomolar concentrations of adrenaline (25$100 \mathrm{nmol} / \mathrm{L})$ required to induce inexcitability within the $\mathrm{PV}_{\text {dis }}$ (determined as a failure of excitation propagation from $\mathrm{PV}_{\text {ost }}$ to $\mathrm{PV}_{\text {dis }}$ under constant LA pacing and APA in $\mathrm{PV}_{\text {dis }}<20 \mathrm{mV}$, see Figure $2 \mathbf{A}$ ). To test if $\mathrm{PV}_{\text {dis }}$ inexcitability was associated with adrenaline-induced RP depolarization rather than microelectrode stability, $1 \mu \mathrm{mol} / \mathrm{L}$ acetylcholine was briefly applied to stimulate the acetylcholine-activated outward potassium current $I_{\mathrm{K}, \mathrm{ACh}}$, hyperpolarize the RP, and recover intra-PV conduction. After that, acetylcholine was washed out, and the next concentration of adrenaline was applied for $10 \mathrm{~min}$. Preparations were constantly paced at stable S1S1 $=300 \mathrm{~ms}$ cycle length. To characterize spontaneous activity within the PV, electrical pacing was stopped for 5-10 min under each experimental condition. The protocol was repeated for $150 \mathrm{mg}$ and $10 \mathrm{~g}$ of weight. Each weight was applied for an hour while adrenaline was washed out. The protocol was about 4$5 \mathrm{~h}$ long and applied to all the preparations tested. Importantly, in all experiments, PV preparations were functionally intact throughout the entire experiment as we demonstrated previously 

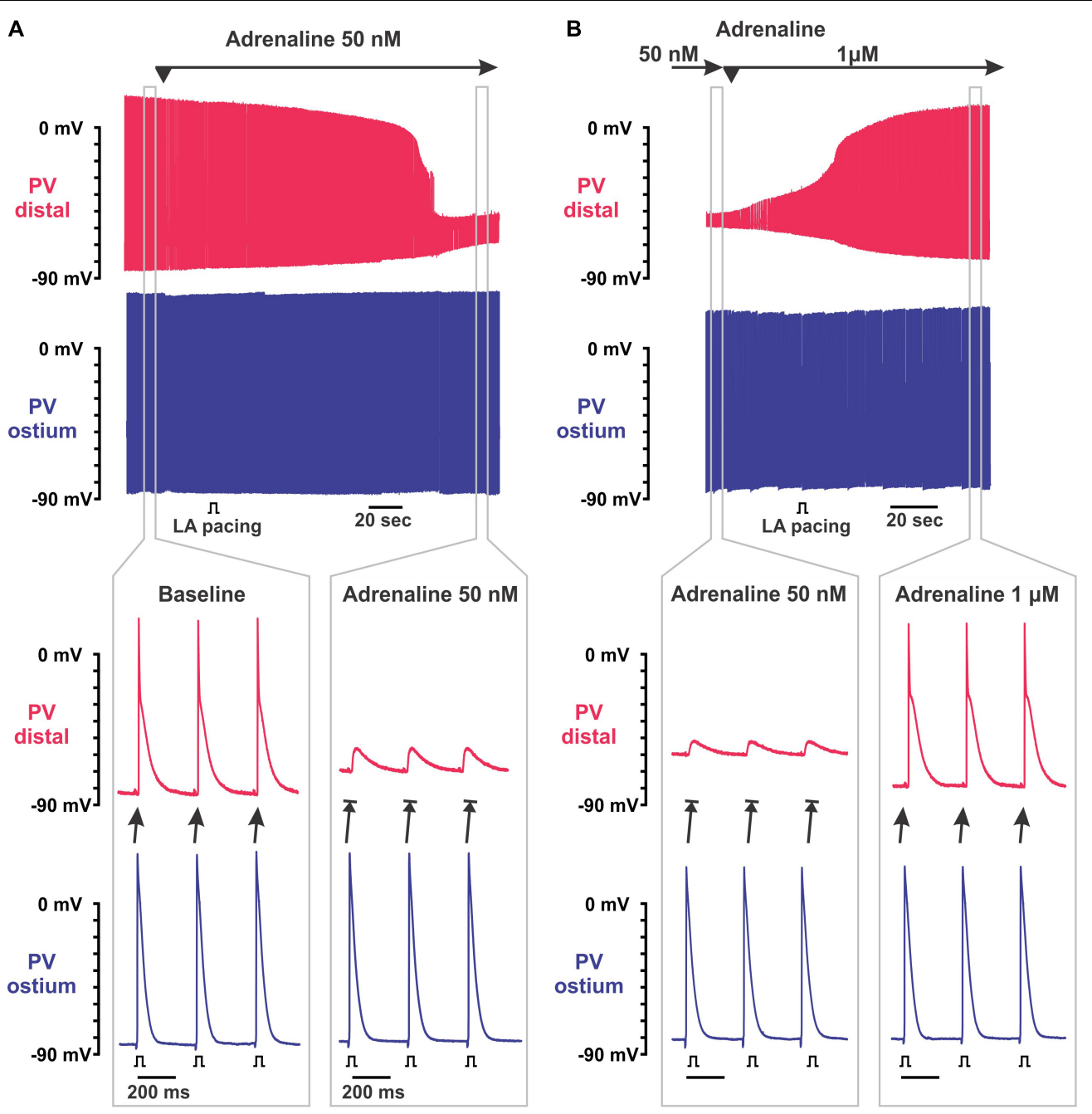

FIGURE 2 | Biphasic effect of adrenaline on intra-pulmonary vein (PV) conduction. (A) Application of $50 \mathrm{nmol} / \mathrm{L}$ adrenaline results in the depolarization of resting membrane potential (RP) exclusively in $\mathrm{PV}_{\text {dis }}$ leading to intra-PV conduction dissociation. Two simultaneous microelectrode recordings from $\mathrm{PV}_{\text {dis }}$ (top recording in red) and $\mathrm{PV}_{\text {ost }}$ (bottom recording in blue) are shown. Below, selected time windows (gray rectangles) are shown enlarged before (baseline) and during $P V_{\text {dis }}$ inexcitability under $50 \mathrm{nmol} / \mathrm{L}$ adrenaline. Left atria (LA) was constantly paced with S1S1 = $300 \mathrm{~ms}$. (B) Application of $1 \mu \mathrm{mol} / \mathrm{L}$ adrenaline recovered intra-PV conduction suppressed by $50 \mathrm{nmol} / \mathrm{L}$ adrenaline. Below, selected time windows (gray rectangles) are shown enlarged before and after the recovery of excitability of $\mathrm{PV}_{\text {dis }}$.

(Egorov et al., 2019) and tested here by their response to regular applications of acetylcholine.

Arrhythmia score was defined according to a type of spontaneous electrical activity event as follows: 0 , no spontaneous activity; 1, an irregular sporadic electrical activity; 2, irregular bursts of fast electrical activity; 3, irregular bursts of fast electrical activity that was suppressed by atrial pacing; 4 , a stable, but slow PV rhythm that could not be suppressed by atrial pacing; and 5, a fast regular PV rhythm that could not be suppressed by atrial pacing and was complicated by echo extra beats.

\section{Statistics}

Student's $t$-test was used in two-group comparisons. Multiple groups of normally distributed data of similar variance were compared by one- or two-way ANOVA. For multiple comparisons, the Bonferroni's corrected $P$ value is shown. All statistical analyses were performed using GraphPad Prism 5 or Origin version 6.1. A value of $P<0.05$ was considered statistically significant. Values were presented as mean \pm SEM.

\section{RESULTS}

Similar to what we have recently demonstrated (Egorov et al., 2019), mild stretch (150 mg) did not affect PV electrophysiology at baseline. Changes in RP, APA, and AP duration (APD) were not observed in both $\mathrm{PV}_{\text {ost }}$ and $\mathrm{PV}_{\text {dis }}$. In contrast, moderate $(10 \mathrm{~g})$ stretch significantly depolarized the RP specifically in $\mathrm{PV}_{\text {dis }}$ ($56 \pm 2 \mathrm{mV}$ vs. $-82 \pm 1 \mathrm{mV}$ at baseline, $P<0.01)$ suppressing $\mathrm{APA}$ and resulting in inexcitability of $\mathrm{PV}_{\text {dis }}$ that was evident from 


\section{A Arrhythmia score} Unstretched

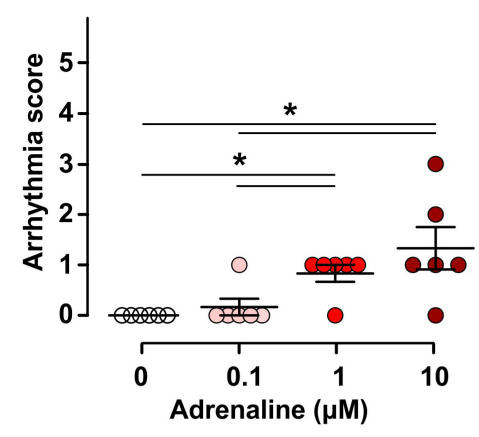

B

$150 \mathrm{mg}$ Stretch, $1 \mu \mathrm{M}$ Adrenaline $\stackrel{\mathrm{NP}}{\stackrel{\mathrm{Adr}}{\longrightarrow} \mathrm{Adr}+\mathrm{ACh}} \underset{\mathrm{S} 1 \mathrm{~S} 1=300 \mathrm{~ms}}{\stackrel{\text { Adr }}{\longrightarrow}}$
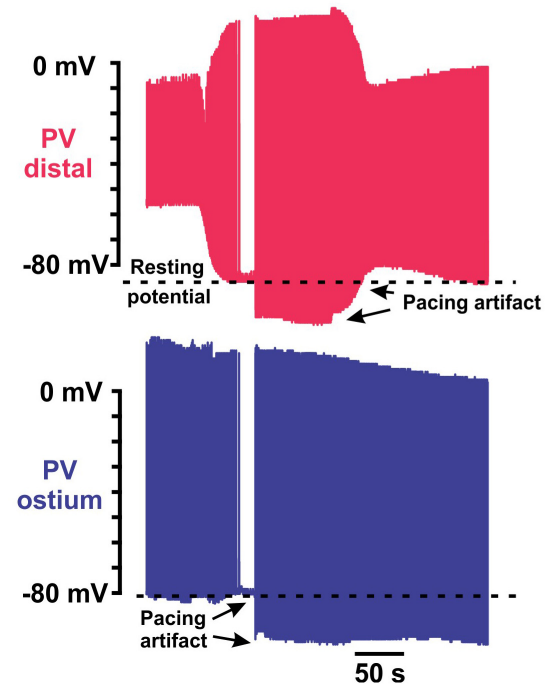

$150 \mathrm{mg}$ stretch

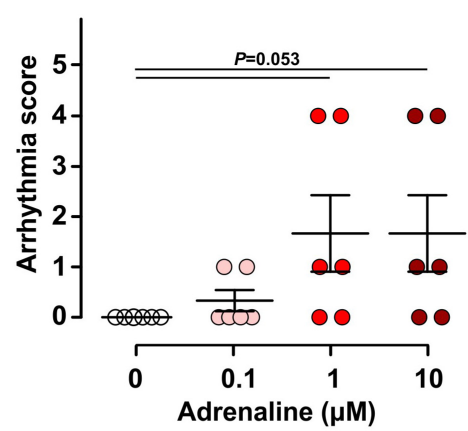

C

Unstretched $10 \mu \mathrm{M}$ Adrenaline
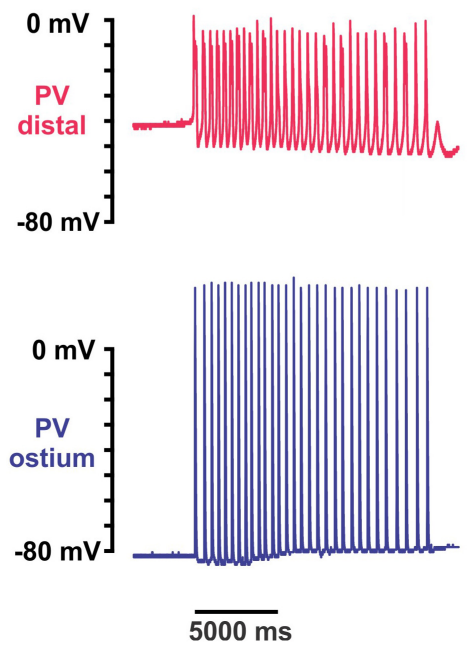

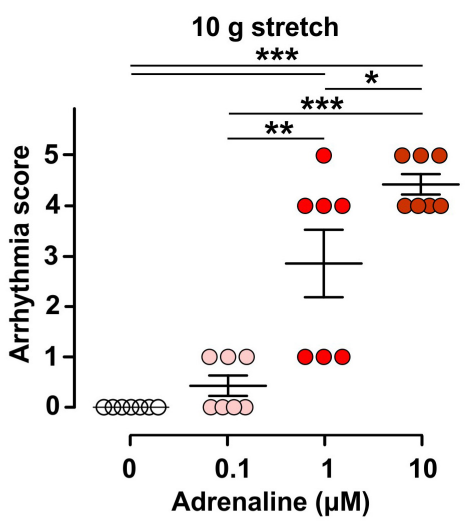

D
$150 \mathrm{mg}$ Stretch
$10 \mu \mathrm{M}$ Adrenaline
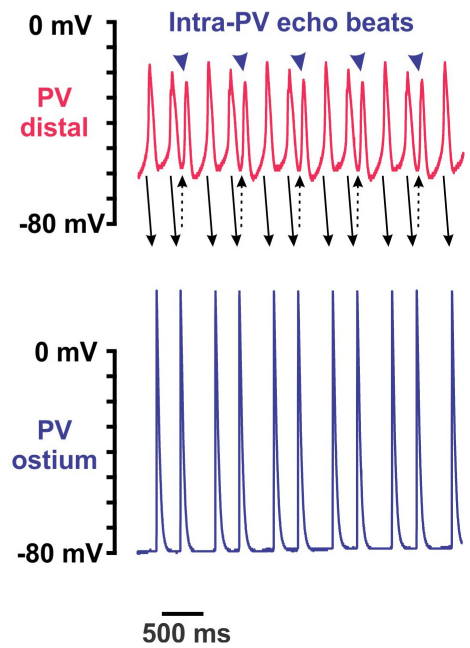

FIGURE 3 | Arrhythmic events induced within the pulmonary vein (PV) myocardium by low (25-100 nmol/L) and high (1-10 $\mu$ mol/L) concentrations of adrenaline in unstretched and stretched (150 mg and 10.5 g) preparations. (A) Arrhythmia score calculated as described in the section "Materials and Methods." $N=6-7$ rats. $P$ values are calculated by repeated measurements two-way ANOVA with Bonferroni correction. (B-D) Representative examples of arrhythmic events recorded in PV preparations under different conditions. Two simultaneous microelectrode recordings from $\mathrm{PV}_{\text {dis }}$ (top recording in red) and $\mathrm{PV}_{\text {ost }}$ (bottom recording in blue) are shown for each event. (B) A brief application of $1 \mu \mathrm{mol} / \mathrm{L}$ acetylcholine (ACh) in $150 \mathrm{mg}$ stretched preparation hyperpolarized the RP in PV dis back to a baseline level and suppressed spontaneous PV activity (no pacing, NP) under $1 \mu \mathrm{mol} / \mathrm{L}$ adrenaline (Adr) application. After ACh washout, spontaneous slow PV rhythm (arrhythmia score is 3) was suppressed by atrial pacing $(\mathbf{S 1 S 1}=300 \mathrm{~ms})$. (C,D) Stable slow rhythm (arrhythmia score is $4, \mathbf{C}$ ) and fast regular rhythm (arrhythmia score is 5 , D) induced by $10 \mu \mathrm{mol} / \mathrm{L}$ adrenaline in unstretched (C) and $150 \mathrm{mg}$ stretched (D) PV preparations.

a failure of the propagation of excitation toward $\mathrm{PV}_{\text {dis }}$ (Figure 1). In $\mathrm{PV}_{\text {ost }}$, neither RP or APA were changed at moderate stretch, while APD was significantly prolonged from $55 \pm 2 \mathrm{~ms}$ to $84 \pm 8$ ms, respectively $(P<0.01)$.

Low concentrations of adrenaline (25-100 nmol/L) depolarized the RP selectively within $\mathrm{PV}_{\text {dis }}$ (by $26 \pm 2 \mathrm{mV}$ at baseline and $18 \pm 1 \mathrm{mV}$ at mild stretch, $P<0.001)$ and did not change the $\mathrm{RP}$ in $\mathrm{PV}_{\text {ost }}$ measured during a stable atrial stimulation of S1S1 = $300 \mathrm{~ms}$ (Figure 1). This subsequently suppressed an APA in $\mathrm{PV}_{\text {dis }}$ and resulted in the development of intra-PV conduction dissociation (Figure 2A). At moderate stretch, this further depolarized the RP in the setting of $\mathrm{PV}_{\mathrm{dis}}$ inexcitability by $5.9 \pm 1.1 \mathrm{mV}$ at $10 \mathrm{~g}, P<0.01$. A brief application of acetylcholine hyperpolarized the RP back to baseline values and successfully recovered $\mathrm{PV}_{\text {dis }}$ excitability in all preparations (Figure 3B).

Subsequent application of $1 \mu \mathrm{mol} / \mathrm{L}$ adrenaline recovered $\mathrm{PV}_{\text {dis }}$ excitability and the intra-PV conduction in all the preparations tested (see representative example in Figure 2B). In unstretched and $150 \mathrm{mg}$ stretched preparations, it was associated with significant RP hyperpolarization and recovery of the APA $(P<0.01)$ in $\mathrm{PV}_{\text {dis }}$. In contrast, in $10 \mathrm{~g}$ stretched preparations, 


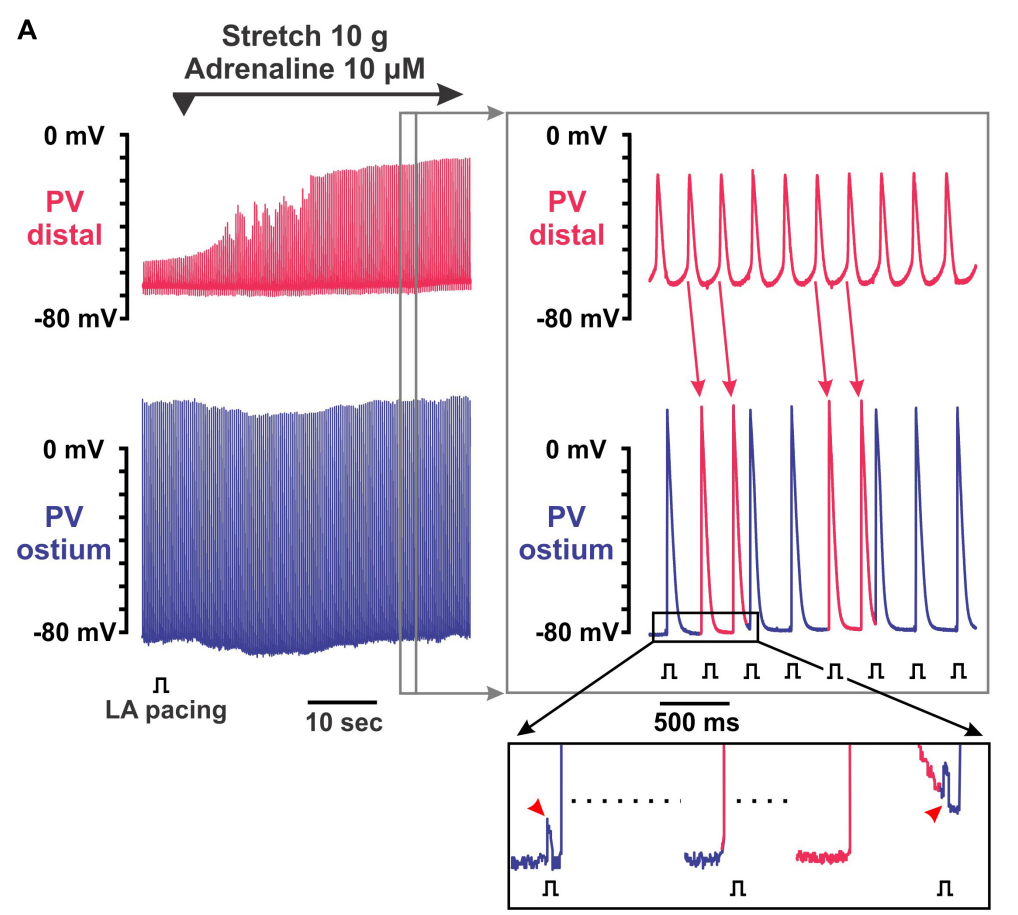

\section{B \\ B Stretch $10 \mathrm{~g}$ \\ Adrenaline $10 \mu \mathrm{M}$}
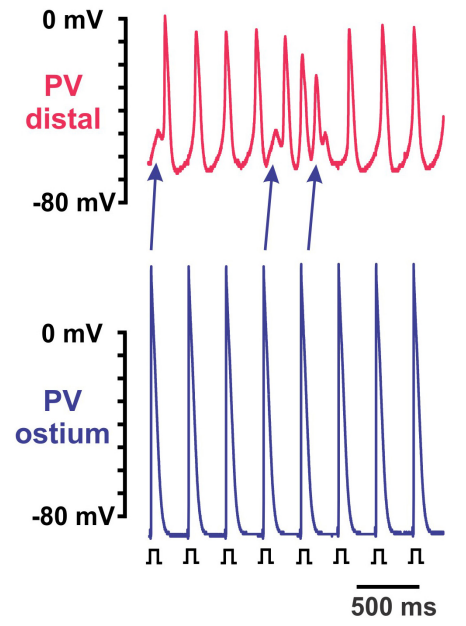

FIGURE 4 | Complex electrical activity recorded in pulmonary vein (PV) preparations under $10 \mu \mathrm{mol} / \mathrm{L}$ adrenaline applied during $10 \mathrm{~g}$ stretch. Two simultaneous microelectrode recordings from PV distal (top recording in red) and PV ostium (bottom recording in blue) are shown. (A) Recovery of intra-PV conduction with arrhythmogenic activity after application of $10 \mu \mathrm{mol} / \mathrm{L}$ adrenaline in $10 \mathrm{~g}$ stretched PV. Left panel shows a continuous 1-min recording of adrenaline application, while right panel represents an enlarged section of the left recording indicated by a gray rectangle. Spontaneous activity was induced in distal PV and not suppressed by left atrial (LA) pacing. Instead, a faster rhythm in distal PV interfered with a slower rate of electrical pacing resulting in frequent atrial extra beats (shown as red action potentials in PV ostium recordings; red arrows indicate propagating PV distal beats). (B) Burst of fast electrical activity triggered in distal PV by atrial extra beat interfered with intrinsic PV rhythm.

recovery of the intra-PV conduction was not associated with changes in the RP and only partial recovery of the APA in $\mathrm{PV}_{\text {dis }}$ (Figure 1). In $\mathrm{PV}_{\mathrm{ost}}$, neither RP or APA were changed under 1 and $10 \mu \mathrm{mol} / \mathrm{L}$ adrenaline in both mildly and moderately stretched preparations.

Although 25-100 nmol/L adrenaline could strongly depolarize the $\mathrm{RP}$ in $\mathrm{PV}_{\text {dis }}$, this was not associated with the induction of automatic activity, both in unstretched and stretched preparations (arrhythmia index of $0.17 \pm 0.17, P=0.340$ in unstretched preparations, $0.33 \pm 0.21, P=0.145$, and $0.43 \pm 0.20$, $P=0.055$, in $150 \mathrm{mg}$ and $10 \mathrm{~g}$ stretched preparations, respectively; no spontaneous activity was observed without adrenaline; Figure 3A). This was opposite to higher concentrations of adrenaline (1-10 $\mu \mathrm{mol} / \mathrm{L})$, which evoked spontaneous activity in all preparations studied, with (unstretched and 150-mg stretched) and without (10-g stretched) concomitant hyperpolarization of the RP (Figures 3C,D). Moderate stretch significantly facilitated the triggering of rapid PV firing by adrenaline (arrhythmia index: $4.4 \pm 0.2$ vs. $1.3 \pm 0.4$ in unstretched, $P<0.001$, and $1.7 \pm 0.8$ in mildly stretched preparations, $P<0.005$, at $10 \mu \mathrm{mol} / \mathrm{L}$ adrenaline) and induced frequent episodes of intra-PV "echo" beats (Figure 3D) (Egorov et al., 2015; Bun et al., 2018).

Spontaneous activity induced by adrenaline in moderately stretched preparations were characterized by complex interactions between $\mathrm{PV}_{\text {dis }}$ and $\mathrm{PV}_{\text {ost }}$. Figure $4 \mathrm{~A}$ shows an example of the initiation of PV spontaneous activity by application of $10 \mu \mathrm{mol} / \mathrm{L}$ adrenaline in a $10 \mathrm{~g}$ stretched preparation that led to irregular atrial extra beats. This activity was not suppressed by LA pacing. Instead, a faster rhythm in $\mathrm{PV}_{\text {dis }}$ interfered with a slower rate of electrical pacing resulting in frequent atrial extra beats (shown in Figure 4A as red APs in $\mathrm{PV}_{\mathrm{ost}}$ recordings). Atrial extra beats triggered from $\mathrm{PV}_{\mathrm{dis}}$ had different morphology as shown in the enlarged insert below: while paced APs (blue in $\mathrm{PV}_{\text {ost }}$ recording) followed pacing artifact, triggered extra beats (red in $\mathrm{PV}_{\text {ost }}$ recording) were not synchronized with atrial pacing and not associated with pacing artifacts. Another example shown in Figure 4B demonstrates how atrial beats can initiate arrhythmogenic burst of fast electrical activity in $\mathrm{PV}_{\mathrm{dis}}$. Altogether, these findings show how spontaneous activity induced by sympathetic stimulation and facilitated by PV stretch can interfere with a stable atrial rhythm resulting in potentially arrhythmogenic atrial extra beats that can initiate atrial fibrillation.

\section{DISCUSSION}

Our findings demonstrate complex interactions between the sympathetic tone and mechanical stretch in the development of arrhythmogenic activity within PVs. First, we found a 
biphasic effect of adrenaline on PVs; while low concentrations of adrenaline (25-100 nmol/L) depolarized the RP and led to intra-PV conduction dissociation, high doses of adrenaline (1$10 \mu \mathrm{mol} / \mathrm{L})$ led to RP hyperpolarization and the development of spontaneous electrical potentials within the PV. This biphasic effect of adrenaline could be linked to a dose-dependent activation of predominantly $\alpha$ - at low concentrations and both $\alpha$ - and $\beta$-adrenergic receptors at high concentrations (Furchgott, 1967). It was previously shown that stimulation of $\beta$-adrenergic receptors decreases the membrane stabilizing outward current $I_{\mathrm{K} 1}$ (Braun et al., 1992) and enhances the volume-regulated inward chloride current $I_{\mathrm{Cl} \text {,swell }}$ (Ellershaw et al., 2002) that, together, would lead the RP depolarization as observed here and previously by Doisne et al. (2009). In contrast, stimulation of $\beta$-adrenergic receptors by high concentrations of adrenaline would increase $I_{\mathrm{K} 1}$ but decrease $I_{\mathrm{Cl} \text {,swell }}$, hyperpolarizing the RP. Importantly, all these effects were observed under a stable atrial pacing when PV RP did not differ from that measured in the left ventricle as shown here (Figure 1) and previously (Doisne et al., 2009; Egorov et al., 2015).

We also found that pathological (moderate) stretch facilitated the development of arrhythmogenic ectopic activity induced by high concentrations of adrenaline in PVs. At depolarized RPs under stretch, application of adrenaline led to more regular and faster PV automaticity compared with nonstretched conditions (Figure 3A). Interestingly, although both moderate stretch and low concentrations of adrenaline significantly depolarized the $\mathrm{RP}$, those were not associated with the induction of automatic activity and required an application of high concentrations of adrenaline. This indicate a crucial combination of pathologically depolarized RP and enhanced $\mathrm{Ca}^{2+}$ handling to form both a substrate [at cellular as well as tissue levels (Egorov et al., 2019)] and a trigger [in a form of early afterdepolarizations (Patterson et al., 2005)] for a stable spontaneous activity within PVs. As it was shown by Patterson et al. (2005), suppression of ryanodine receptor activity (by ryanodine) or reduction in the transmembrane gradient driving $\mathrm{Na} / \mathrm{Ca}$ exchange (by increase $\left[\mathrm{Ca}^{2+}\right]_{\mathrm{o}}$ from 1.35 to $5 \mathrm{mM}$ ) completely suppressed spontaneous firing from PVs, without changed in RP.

Importantly, spontaneous electrical activity induced in the stretched PV by adrenaline was not suppressed by atrial pacing. Moreover, it led to frequent atrial extra beats that may potentially trigger arrhythmogenic atrial extra beats and thus initiate atrial

\section{REFERENCES}

Arora, R., Verheule, S., Scott, L., Navarrete, A., Katari, V., Wilson, E., et al. (2003). Arrhythmogenic substrate of the pulmonary veins assessed by high-resolution optical mapping. Circulation. 107, 1816-1821.

Braun, A. P., Fedida, D., and Giles, W. R. (1992). Activation of alpha 1adrenoceptors modulates the inwardly rectifying potassium currents of mammalian atrial myocytes. Pflugers. Arch. 421, 431-439.

Bun, S. S., Latcu, D. G., Wedn, A. M., and Saoudi, N. (2018). Intrapulmonary vein "echo" beats'. HeartRhythm Case Rep. 4, 464-465.

Chen, S. A., Hsieh, M. H., Tai, C. T., Tsai, C. F., Prakash, V. S., Yu, W. C., et al. (1999). Initiation of atrial fibrillation by ectopic beats originating from the pulmonary veins: electrophysiological characteristics, pharmacological responses, and effects of radiofrequency ablation. Circulation. 100, 1879-1886. fibrillation. It should be also noted that myocardium dilation during pressure and/or volume overload of the atria could result in heterogeneous distribution of wall stress creating regions of conduction slowing and thus facilitating the induction of atrial fibrillation by PV extra beats. Altogether, our findings highlight an arrhythmogenic impact of PV stretch in the development of atrial arrhythmias under elevated autonomic tone, which could play a critical role in patients with elevated blood pressure associated with hypertension, heart failure, and valvular disease.

\section{DATA AVAILABILITY STATEMENT}

The datasets generated for this study are available on request to the corresponding author.

\section{ETHICS STATEMENT}

The animal study was reviewed and approved by the Animal Care and Use Committee of the Cardiology Research Center (Moscow, Russia).

\section{AUTHOR CONTRIBUTIONS}

YE, LR, and AG substantially contributed to the conception and design of the work; the acquisition, analysis, or interpretation of the data and literature; drafting of the work critically for important intellectual content; providing of approval for publication of the content; and agreeing to be accountable for all aspects of the work in ensuring that questions related to the accuracy or integrity of any part of the work are appropriately investigated and resolved.

\section{FUNDING}

This work was supported by the Russian Foundation for Basic Research Grants 17-04-01634 and Scientific Research Work AAAA-A18-118022290082-7 (to LR), and NIH 1R01HL14121401, AHA 16SDG29120011 and the Wisconsin Partnership Program (to AG).

Doisne, N., Maupoil, V., Cosnay, P., and Findlay, I. (2009). Catecholaminergic automatic activity in the rat pulmonary vein: electrophysiological differences between cardiac muscle in the left atrium and pulmonary vein. Am. J. Physiol. Heart. Circ. Physiol. 297, H102-H108. doi: 10.1152/ajpheart.00256. 2009

Egorov, Y. V., Kuz'min, V. S., Glukhov, A. V., and Rosenshtraukh, L. V. (2015). Electrophysiological Characteristics, Rhythm, Disturbances and Conduction Discontinuities Under Autonomic Stimulation in the Rat Pulmonary Vein Myocardium. J. Cardiovasc. Electrophysiol. 26, 1130-1139. doi: 10.1111/jce. 12738

Egorov, Y. V., Lang, D., Tyan, L., Turner, D., Lim, E., Piro, Z. D., et al. (2019). Caveolae-Mediated Activation of Mechanosensitive Chloride Channels in Pulmonary Veins Triggers Atrial Arrhythmogenesis. J. Am. Heart. Assoc. 8, e012748. doi: 10.1161/JAHA.119.012748 
Ellershaw, D. C., Greenwood, I. A., and Large, W. A. (2002). Modulation of volume-sensitive chloride current by noradrenaline in rabbit portal vein myocytes. J. Physiol. 542, 537-547.

Furchgott, R. F. (1967). The pharmacological differentiation of adrenergic receptors. Annals of the New York Academy of Sciences. 139, 553-570.

Haissaguerre, M., Jais, P., Shah, D. C., Takahashi, A., Hocini, M., Quiniou, G., et al. (1998). Spontaneous initiation of atrial fibrillation by ectopic beats originating in the pulmonary veins. N. Engl. J. Med. 339, 659-666.

Malecot, C. O., Bredeloux, P., Findlay, I., and Maupoil, V. (2015). A TTX-sensitive resting $\mathrm{Na}+$ permeability contributes to the catecholaminergic automatic activity in rat pulmonary vein. J. Cardiovasc. Electrophysiol. 26, 311-319. doi: $10.1111 /$ jce. 12572

Melnyk, P., Ehrlich, J. R., Pourrier, M., Villeneuve, L., Cha, T. J., and Nattel, S. (2005). Comparison of ion channel distribution and expression in cardiomyocytes of canine pulmonary veins versus left atrium. Cardiovasc. Res. $65,104-116$

Okamoto, Y., Kawamura, K., Nakamura, Y., and Ono, K. (2014). Pathological impact of hyperpolarization-activated chloride current peculiar to rat pulmonary vein cardiomyocytes. J. Mol. Cell. Cardiol. 66, 53-62. doi: 10.1016/j. yjmcc.2013.11.002

Pasqualin, C., Yu, A., Malecot, C. O., Gannier, F., Cognard, C., Godin-Ribuot, D., et al. (2018). Structural heterogeneity of the rat pulmonary vein myocardium: consequences on intracellular calcium dynamics and arrhythmogenic potential. Sci. Rep. 8, 3244. doi: 10.1038/s41598-018-21671-9

Patterson, E., Po, S. S., Scherlag, B. J., and Lazzara, R. (2005). Triggered firing in pulmonary veins initiated by in vitro autonomic nerve stimulation. Heart Rhythm. 2, 624-631.
Schotten, U., Verheule, S., Kirchhof, P., and Goette, A. (2011). Pathophysiological mechanisms of atrial fibrillation: a translational appraisal. Physiol. Rev. 91, 265-325. doi: 10.1152/physrev.00031.2009

Tsao, H. M., Yu, W. C., Cheng, H. C., Wu, M. H., Tai, C. T., Lin, W. S., et al. (2001). Pulmonary vein dilation in patients with atrial fibrillation: detection by magnetic resonance imaging. J. Cardiovasc. Electrophysiol. 12, 809-813.

Tsuneoka, Y., Irie, M., Tanaka, Y., Sugimoto, T., Kobayashi, Y., Kusakabe, T., et al. (2017). Permissive role of reduced inwardly-rectifying potassium current density in the automaticity of the guinea pig pulmonary vein myocardium. J. Pharmacol. Sci. 133, 195-202. doi: 10.1016/j.jphs.2016.12.006

Walters, T. E., Lee, G., Spence, S., Larobina, M., Atkinson, V., Antippa, P., et al. (2014). Acute atrial stretch results in conduction slowing and complex signals at the pulmonary vein to left atrial junction: insights into the mechanism of pulmonary vein arrhythmogenesis. Circ. Arrhythm. Electrophysiol. 7, 11891197. doi: 10.1161/CIRCEP.114.001894

Conflict of Interest: The authors declare that the research was conducted in the absence of any commercial or financial relationships that could be construed as a potential conflict of interest.

Copyright (c) 2020 Egorov, Rosenshtraukh and Glukhov. This is an open-access article distributed under the terms of the Creative Commons Attribution License (CC BY). The use, distribution or reproduction in other forums is permitted, provided the original author(s) and the copyright owner(s) are credited and that the original publication in this journal is cited, in accordance with accepted academic practice. No use, distribution or reproduction is permitted which does not comply with these terms. 